\title{
Analysis and optimization of a free-electron laser with an irregular waveguide
}

\author{
V. A. Goryashko* \\ Institute for Radiophysics and Electronics of NAS of Ukraine, 12 Academician Proskura Street, Kharkiv 61085, Ukraine
}

(Received 2 April 2010; published 8 March 2011)

\begin{abstract}
Using a time-dependent approach, the analysis and optimization of a planar free-electron laser (FEL) amplifier with an axial magnetic field and an irregular waveguide is performed. By applying methods of nonlinear dynamics, a self-consistent reduced model of the FEL is built in a special phase space. This reduced model is the generalization of the Colson-Bonifacio model and takes into account the intricate dynamics of electrons in the pump magnetic field and the intramode scattering in the irregular waveguide. The reduced model and concepts of evolutionary computation are used to find optimal waveguide profiles. The numerical simulation of the original nonsimplified model is performed to check the effectiveness of found optimal profiles. To demonstrate advantages of the proposed FEL configuration, the parameters are chosen to be close to the parameters of the experiment [S. Cheng et al., IEEE Trans. Plasma Sci. 24, 750 (1996)], in which a sheet electron beam with the moderate thickness interacts with the $\mathrm{TE}_{01}$ mode of a rectangular waveguide. The results strongly indicate that one can improve the efficiency by a factor of 5 or 6 if the FEL operates in the magnetoresonance regime and if the irregular waveguide with the optimized profile is used. The FEL efficiency is maximal if the initial beam energy is slightly higher than the energy that corresponds to a transition between negative- and positive-mass regimes so that the transition from the negative-mass to positive-mass regimes occurs during the beam-wave interaction.
\end{abstract}

DOI: 10.1103/PhysRevSTAB.14.030703

PACS numbers: 41.60.Cr, 05.45.-a, 84.40.Ik, 52.59.Sa

\section{INTRODUCTION}

The progress in physics and technology of generation of electron beams makes possible emission and transport of superwide sheet beams with peak power up to several tens of gigawatts. For example, in the paper [1] emission and transport of a $140 \mathrm{~cm}$-wide, $20 \mathrm{kA}$ and $2 \mathrm{MeV}$ sheet electron beam for purported free-electron laser applications was reported. Other promising results on generation of two intense beams have been recently reported in [2]: $0.8 \mathrm{MeV}$ electron energy, current densities of up to $1.5 \mathrm{kA} / \mathrm{cm}^{2}$, $0.4 \times 7.0 \mathrm{~cm}^{2}$ beam cross sections. It is an attractive idea to convert such huge beam power into radiation and this can be done using free-electron lasers (FEL) in their planar configuration. However, the efficiency of FELs is typically small if there is no optimization. In this paper we will consider possible ways to increase the FEL efficiency by applying an axial magnetic field and using a waveguide with an optimized profile. Recently, interest toward FELs for plasma fusion has been reinforced [3] and a high-power planar FEL seems to be a promising source when multimegawatt millimeter radiation is required. In fact, gyrotrons are used for plasma heating in some operating tokamaks and stellarators, but these setups are quite small and a few gyrotrons provide sufficient power. However, to obtain a

\footnotetext{
*vitgor@ire.kharkov.ua
}

Published by American Physical Society under the terms of the Creative Commons Attribution 3.0 License. Further distribution of this work must maintain attribution to the author(s) and the published article's title, journal citation, and DOI. positive energy output, larger setups are necessary and the required quasi-CW microwave power for the ITER project is about $100 \mathrm{MW}$ so that the FEL with a sheet electron beam might be a reasonable alternative.

It is worth noting that, in vacuum electronic sources of coherent radiation, the electron beams are far from the statistical equilibrium and during their interaction with radiation they remain sufficiently nonequilibrium $[4,5]$. Thus, the efficiency of the transfer of the electrons' kinetic energy into radiation, basically, may be close to $100 \%$ (the klystron or traveling wave tube are the examples of high-efficiency devices) and the challenge is to optimize the beam-wave interaction by controlling the most important parameters. There are several ways to improve the FEL efficiency: optimization of electron beam characteristics (for example, development of beams with an optimal correlated energy spread when the effect of the beam finite thickness is relevant), tapering of the undulator or the axial magnetic field, and profiling of waveguide/resonator walls. In particular, the effectiveness and reliability of the undulator tapering were demonstrated theoretically [6,7] and confirmed experimentally [8] to a great advantage. A high effectiveness of the undulator tapering was also demonstrated for a FEL with an axial magnetic field [9]. At the same time there exist cases where the convenient undulator profiling cannot be used or ensure desired enhancement. In particular, if an electromagnetic wave undulator is used, then, clearly, one has to optimize characteristics of an electrodynamic structure. The optimization of the electromagnetic structure also seems to be more efficient than the undulator profiling if the effect of the beam finite thickness is relevant. 
In the present paper, I demonstrate that one can effectively suppress beam layering and the saturation efficiency effect by using the optimized profiled waveguide. This technique may be useful for the development of highpower planar FELs with finite-thickness sheet beams. This paper is structured as follows: in Sec. II the problem statement for the planar FEL amplifier with the axial magnetic field and the irregular waveguide is defined. The nonlinear dynamics of a test electron in the pump magnetic field is analyzed in Sec. III. A self-consistent reduced model of the FEL is formulated in Sec. IV and its features are discussed. In the following section, principles of the beam-wave control are considered and a practical example of the optimized FEL is given. The obtained results are discussed in the Summary, and, finally, the time-dependent excitation equation of the irregular waveguide and conditions of the internal nonlinear resonances are derived in Appendices A and B, respectively.

\section{THE THEORETICAL MODEL}

Let a sheet relativistic electron beam be injected into an irregular waveguide located in the external pump magnetic field that consists of the magnetic field of a linearly polarized (planar) undulator and a uniform axial magnetic field (see Fig. 1). The pump magnetic field is given by the vector potential:

$$
A_{x}^{p}(\vec{r})=\left(B_{u} / k_{u}\right) \cosh \left(k_{u} y\right) \cos \left(k_{u} z\right)+B_{\|} y .
$$

Here $B_{\|}$is the uniform axial guide field, $B_{u}$ is the magnitude of the planar undulator field [10], and $k_{u}=2 \pi / \lambda_{u}$ and $\lambda_{u}$ are the wave number and the period of the undulator, respectively. In simulations the first six periods of the undulator are tapered to introduce the beam adiabatically into the regular section of the undulator [11]. The unmodulated electron beam enters the interaction region, $z \in[0, L]$, with mean longitudinal velocity $V_{\|}$. The irregular waveguide boundaries are set by expressions: $x= \pm a / 2$ and $y= \pm w(z) / 2(a \gg w)$, where $w(z)$ describes the varying distance between two wide walls of the waveguide, and $w^{\prime}(0)=w^{\prime}(L)=0$. Let the FEL amplifier be seeded by the $\mathrm{TE}_{01}$ mode, which is resonant (synchronous) with the electron beam, the mode frequency,

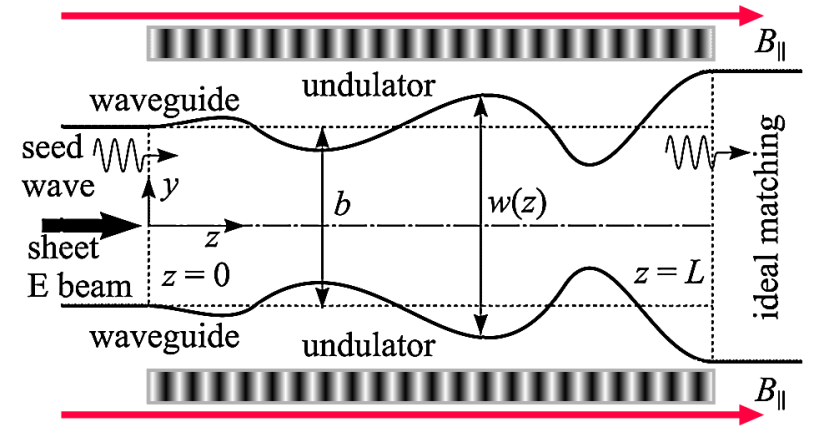

FIG. 1. Sketch of the FEL in the $x=0$ cross section. and the amplitude at the input into the interaction region $(z=0)$ equaling $\omega$ and $V_{0}$, respectively. We consider that the interaction region is ideally matched to the regular output waveguide at the section $z=L$.

Since the wide walls are profiled, $y= \pm w(z) / 2$, one can apply the local Fourier-series expansion [12] over $y$ to derive a coupled set of equations governing the evolution of the $\mathrm{TE}_{n m}$ and $\mathrm{TM}_{n m}$ modes (subscripts $n$ and $m$ correspond to field variations along the wide and narrow walls, respectively). Modes with odd $\mathrm{TE}_{n, \text { odd }}, \mathrm{TM}_{n \text {,odd }}$ and even $\mathrm{TE}_{n, \text { even }}, \mathrm{TM}_{n \text {,even }}$ variations are not coupled because of the waveguide symmetry with respect to the $x z$ plane. We will hold that $\lambda>2 w(z) / 3$, and the $\mathrm{TE}_{0 m}$ modes for $m>3$ are then evanescent so that the scattering of the seed $\mathrm{TE}_{01}$ mode to those modes as well as the backward rescattering from $\mathrm{TE}_{0 m}$ to $\mathrm{TE}_{01}$ will be neglected. This implies that we will ignore the intermode scattering, but take into account the intramode one. We will also ignore the excitation of parasitic modes like $\mathrm{TE}_{n 1}$ and $\mathrm{TM}_{n 1}$ because their resonant frequencies are different from the $\mathrm{TE}_{01}$ mode resonant frequency (see discussion of this point in Sec. V). Under these assumptions the evolution of the signal $\mathrm{TE}_{01}$ mode is governed by the $x$ component of vector potential $A_{x}^{s}$ :

$$
A_{x}^{s}(\vec{r}, t)=\operatorname{Re}\left\{V(z, t) \sqrt{\frac{b}{w(z)}} \cos \left(\frac{\pi y}{w(z)}\right) e^{-i \omega t}\right\} .
$$

Here $V(z, t)$ is the slow-in-time amplitude satisfying the equation (see Appendix A)

$$
\begin{aligned}
& \left\{\frac{\partial^{2}}{\partial z^{2}}+k_{z}^{2}+\frac{2 i k_{z}}{v_{g r}} \frac{\partial}{\partial t}\right\} V(z, t) \\
& =-\frac{8 \omega}{S c} \sqrt{\frac{b}{w(z)}} \int_{-a / 2}^{a / 2} d x \int_{-w(z) / 2}^{w(z) / 2} d y \\
& \quad \times \int_{t-\pi / \omega}^{t+\pi / \omega} d t^{\prime} j_{x}\left(\vec{r}, t^{\prime}\right) \cos \left(\frac{\pi y}{w(z)}\right) e^{i \omega t^{\prime}}
\end{aligned}
$$

where

$$
k_{z}^{2}(z)=\frac{\omega^{2}}{c^{2}}-\left(\frac{\pi}{w(z)}\right)^{2}-\left(\frac{w^{\prime}(z)}{2 w(z)}\right)^{2}\left[1+\frac{\pi^{2}}{3}\right]
$$

is the wave number squared, $v_{g r}(z, \omega)=\left(d k_{z} / d \omega\right)^{-1}$ is the group velocity, $c$ is the speed of light, and $S=a \times b$. The boundary conditions read

$$
\begin{aligned}
& \left.\left(\frac{\partial V}{\partial z}+i k_{z} V-\frac{1}{v_{g r}} \frac{\partial V}{\partial t}\right)\right|_{z=0}=2 i k_{z} V_{0}, \\
& \left.\left(\frac{\partial V}{\partial z}-i k_{z} V+\frac{1}{v_{g r}} \frac{\partial V}{\partial t}\right)\right|_{z=L}=0 .
\end{aligned}
$$

The microscopic current density is given by the following sum over electron trajectories [11]: 


$$
\begin{aligned}
\vec{j}(\vec{r}, t)= & \frac{I_{0}}{S_{b}} \int_{-X_{b} / 2}^{X_{b} / 2} d x_{0} \int_{-Y_{b} / 2}^{Y_{b} / 2} d y_{0} \\
& \times \int_{t-L / V_{\|}}^{t} d t_{e} \frac{\vec{p}\left(z ; \vec{r}_{\perp 0}, t_{e}\right)}{p_{z}\left(z ; \vec{r}_{\perp 0}, t_{e}\right)} \\
& \times \delta\left[\vec{r}_{\perp}-\vec{r}_{\perp}\left(z ; \vec{r}_{\perp 0}, t_{e}\right)\right] \delta\left[t-t\left(z ; \vec{r}_{\perp 0}, t_{e}\right)\right],
\end{aligned}
$$

where $I_{0}$ is the beam current at the input into the interaction region; $S_{b}=X_{b} Y_{b}$ is the cross sectional area of the beam; $\vec{p}\left(z ; \vec{r}_{\perp 0}, t_{e}\right)$ and $\vec{r}_{\perp}\left(z ; \vec{r}_{\perp 0}, t_{e}\right)$ are the mechanical momentum and the transverse coordinate, respectively; $t\left(z ; \vec{r}_{\perp 0}, t_{e}\right)$ is the arrival time of an electron at the position $z ; t_{e}$ and $\vec{r}_{\perp 0}=\vec{r}_{\perp 0}\left(x_{0}, y_{0}\right)$ are the entrance time and the transverse coordinates, which the electron has at the input of the interaction region. The sheet electron beam is lying from $x_{0}=-X_{b} / 2$ to $x_{0}=X_{b} / 2$ and from $y_{0}=-Y_{b} / 2$ to $y_{0}=Y_{b} / 2$ in the $x$ and $y$ directions, respectively. Since the relativistic electron-wave interaction is being studied, the nonradiated fields (space-charge fields) are supposed to be negligible. The relativistic effects result in that the defocusing influence of the potential electric field is partially suppressed by the rotational nonradiated electric and magnetic fields (see [13] for details).

The motion of a typical electron within the electron beam can be described by the relativistic Hamiltonian:

$$
\mathcal{H}=\sqrt{m_{e}^{2} c^{4}+\left(c \vec{P}-e \vec{A}^{p}-e \vec{A}^{s}\right)^{2}}=m_{e} \gamma c^{2} .
$$

Here $e$ and $m_{e}$ are the electron charge and rest mass, respectively; the canonical momentum $\vec{P}$ is related to the mechanical momentum $\vec{p}$ by $\vec{P}=\vec{p}+(e / c)\left(\vec{A}^{p}+\vec{A}^{s}\right)$. The initial conditions for the mechanical momentum and coordinates read

$$
\begin{aligned}
\left.p_{x}\right|_{t=t_{e}} & =\left.p_{y}\right|_{t=t_{e}}=0,\left.\quad p_{z}\right|_{t=t_{e}}=\mathcal{E} V_{\|} / c^{2}, \\
\left.x\right|_{t=t_{e}} & =x_{0},\left.\quad y\right|_{t=t_{e}}=y_{0},\left.\quad z\right|_{t=t_{e}}=0,
\end{aligned}
$$

where $\mathcal{E}$ is the initial energy of the electron entering the interaction region at the time $t_{e}$. The field representations (1) and (2) and excitation equation (3) along with the expression for the current density (5) as well as the equations of motion generated by the Hamiltonian (6) describe the electron-wave interaction in the studied FEL in a selfconsistent way.

In order to find parameters and a waveguide profile that provide the maximal efficiency, one has to apply an optimization technique. However, a direct numerical optimization based on the nonaveraged FEL model formulated above fails to work because a vast amount of computational resources is required. Typically, about several thousand equations of motion and the partial differential equation for the wave amplitude have to be simulated. In this paper we propose another approach to the problem. The investigation is divided into several stages: initially equations of motion and the excitation equation are partly integrated in an analytical way using methods of nonlinear dynamics. As a result, a reduced FEL model is derived in a special phase space. Then with this model and some principles of evolutionary computations (genetic algorithms), the numerical optimization of the waveguide profile is performed. Finally, the simulation of the nonsimplified original model using the found optimal waveguide profiles is carried out.

For the subsequent analysis, it is advanced to rewrite Eq. (6) as

$$
\mathcal{H}(\vec{r}, \vec{P}, t)=\sqrt{\overline{\mathcal{H}}^{2}(\vec{r}, \vec{P})+W(\vec{r}, \vec{P}, t)},
$$

where $\overline{\mathcal{H}}$ describes the dynamics of a typical electron in the pump magnetic field,

$$
\begin{aligned}
\bar{H}= & {\left[\left\{c P_{x}-e\left[\left(B_{u} / k_{u}\right) \cosh \left(k_{u} y\right) \cos \left(k_{u} z\right)+B_{\|} y\right]\right\}^{2}\right.} \\
& \left.+\left(c P_{y}\right)^{2}+\left(c P_{z}\right)^{2}+m_{e}^{2} c^{4}\right]^{1 / 2}=m_{e} \gamma_{0} c^{2},
\end{aligned}
$$

and the ponderomotive perturbation reads

$$
W=-2 e\left(c P_{x}-e A_{x}^{p}\right) A_{x}^{s}+\left(e A_{x}^{s}\right)^{2} .
$$

At the beginning of the interaction region $W \approx 0$ and $W$ increases slowly as electrons move through the waveguide and lose their energy to the microwave radiation so that electron trajectories also change. We start our analysis with the integration of the equation of motion generated by the unperturbed Hamiltonian (9) because the electron dynamics in the pump field (1) is fundamental for understanding the FEL operation. The integration of (9) is a nontrivial problem because the nonlinear dynamical system (9) is not globally integrable [14] and exhibits chaotic behavior if the absolute value of the difference between the normal undulator and normal cyclotron frequencies is less than the betatron frequency [13]. The nonlinear dynamics of a test electron in the pump magnetic field is the issue of the next section.

\section{DYNAMICS OF ELECTRONS IN THE PUMP MAGNETIC FIELD}

\section{A. Magnetoresonance and negative-mass regime}

In a special phase space called the action-angle space, the electron motion described by the Hamiltonian (9) is characterized by 2 degrees of freedom, namely, by undulator (subscript $u$ ) and cyclotron (subscript $c$ ) degrees of freedom. Oscillations corresponding to these degrees of freedom can be viewed as normal modes of the nonlinear system (9) and characterized by normal undulator $\Omega_{u}$ and normal cyclotron $\Omega$ frequencies. In the action-angle space, the electron trajectories wind up two-dimensional invariant tori if the dynamics is regular. Examples of such invariant tori and weak disintegration in the case of the ideal pump field can be found in [13]. The transverse inhomogeneous of the realistic undulator field does not lead to appearance of the additional betatron degree of freedom, but only modifies the undulator and cyclotron motion. Note that in 
the pure undulator field $\left(B_{\|}=0\right)$ the cyclotron degree of freedom disappears, but the undulator and betatron degrees of freedom become split and the dimension of the dynamical system is also equal to two [15]. The normal undulator and cyclotron oscillations are complicated nonlinear mixtures of simple partial oscillations in the pure undulator field and pure axial field. These partial oscillations are characterized by partial undulator $\omega_{u}=k_{u} V_{\|}$and cyclotron $\omega_{c}=|e|_{c} B_{\|} / \mathcal{E}$ frequencies. The coupling, $\varepsilon$, between the undulator and the cyclotron oscillations depends on the ratio of the betatron frequency to the undulator one $\varepsilon=$ $\sqrt{2} \omega_{\beta} / \omega_{u}$, where $\omega_{\beta}=|e| c B_{u} /(\sqrt{2} \mathcal{E})$ is the betatron frequency. In what follows we assume that the coupling is quite weak $\varepsilon \ll 1$. Our approximation $\varepsilon \ll 1$ is equivalent to $\mathcal{K} \ll \gamma_{0}^{2}-1$, where $\mathcal{K}=|e| B_{u} \lambda_{u} /\left(2 \pi m_{e} c^{2}\right)$ is the traditional undulator parameter. Then the normal frequencies are given by

$$
\Omega_{u}=x \omega_{u}, \quad \Omega_{c}=\sigma \omega_{u},
$$

where auxiliary parameters $x$ and $\sigma$ satisfy the set of equations

$$
\begin{array}{r}
\frac{\sigma}{\sigma_{0}}=1+\varepsilon^{2}\left(\frac{\left(2 \varkappa^{2}-\sigma^{2}\right) \cosh ^{2}\left[k_{u} y_{0}\right]}{2\left(\varkappa^{2}-\sigma^{2}\right)^{2}}\right. \\
\left.+\frac{\cosh \left[2 k_{u} y_{0}\right]}{4 \sigma^{2}}+\frac{\sinh ^{2}\left[k_{u} y_{0}\right]}{\varkappa^{2}-4 \sigma^{2}}\right), \\
x=1-\frac{\varepsilon^{2} \cosh ^{2}\left[k_{u} y_{0}\right]\left(3 \varkappa^{2}+\sigma^{2}\right)}{4\left(\varkappa^{2}-\sigma^{2}\right)^{2}} .
\end{array}
$$

Here $\sigma_{0}=\omega_{c} / \omega_{u}$ and $y_{0}$ is the initial transverse position of the test electron. The most principal terms of the electron velocity components read

$v_{x}=v_{\perp}\left(\cos \theta_{u}-\cos \theta_{c}\right)-\frac{\bar{v}_{\|} \omega_{\beta}^{2}}{\Omega_{u}^{2}-\Omega_{c}^{2}} \frac{\Omega_{u}}{2 \Omega_{c}} \sinh \left[2 k_{u} y_{0}\right]$,

$v_{y}=v_{\perp}\left(\sin \theta_{c}-\left(\Omega_{c} / \Omega_{u}\right) \sin \theta_{u}\right)$,

$v_{z}=\bar{v}_{\|}+\frac{v_{\perp} \omega_{\beta}}{\sqrt{2}}\left\{\frac{\cos \left[\theta_{u}-\theta_{c}\right]}{\Omega_{u}-\Omega_{c}}+\frac{\cos \left[\theta_{u}+\theta_{c}\right]}{\Omega_{u}+\Omega_{c}}-\frac{\cos 2 \theta_{u}}{2 \Omega_{u}}\right\}$,

where $\theta_{u, c}=\Omega_{u, c}\left(t-t_{e}\right)$ are the undulator and cyclotron phases, the magnitudes of the average axial velocity and the velocity oscillations are

$$
\bar{v}_{\|}=x V_{\|}, \quad v_{\perp}=\frac{\sqrt{2} \bar{v}_{\|} \omega_{\beta} \Omega_{u}}{\Omega_{u}^{2}-\Omega_{c}^{2}} \cosh \left[k_{u} y_{0}\right] .
$$

Note that Eqs. (12) are symmetric in $V_{\|}$as well as $B_{\|}$and yield trajectories, which are independent of the direction of propagation of electrons or the orientation of the axial field. Some details can be also found in [16], pp. 67-68.

As normal undulator frequency $\Omega_{u}$ tends to normal cyclotron frequency $\Omega_{c}$, a resonant enhancement in the magnitudes of the velocities occurs. This well-known situation is called the magnetoresonance and it was early recognized $[16,17]$ that such a resonance results in increase of the FEL gain and efficiency. However, it was found later on $[13,15,18,19]$ that there is no beam-wave interaction if $\Omega_{u}$ is too close to $\Omega_{c}$ because the severe beam degradation and divergence caused by the electron dynamics chaotization occur. The details of chaotization will be discussed below, and now let us pay attention to one more effect that should be taken into account in experimental setups. From Eq. (13) we see $v_{x}$ has the constant component that is nothing but the $\vec{B}_{\|} \times \nabla \vec{B}_{u}$ drift velocity. Because of the drift the distance between narrow waveguide walls has to larger than $X_{b}+2 x\left(L / v_{\|}\right)$, where $X_{b}$ and $L$ are the beam width and the interaction length. We observe that the magnitude of this drift is resonant at $\Omega_{u} \approx \Omega_{c}$, but for group II of regular orbits, $\Omega_{c}>\Omega_{u}$, this effect is not so strong near the magnetoresonance and does not lead to a substantial increase in the waveguide width (see also the discussion in Sec. V and Fig. 11). In the case of a wide sheet beam, the waveguide width is mainly determined by the beam width, but the drift also has to be taken into account.

Let us find approximate solutions to the equation set (12) and obtain explicit expressions for the frequencies and average velocity. We assume that $x \sim 1$ and $\sigma \sim \sigma_{0}$. Then, in the case of $\sigma_{0} \ll 1$ and $\sigma_{0} \gg 1$ we may take $x=1$ and $\sigma_{0}$ in the right-hand sides of Eqs. (12) to obtain the explicit solution. To consider the case $\sigma_{0} \sim 1$, we introduce new small magnitude $\mu=x-\sigma, \mu \ll x$. Neglecting $\mu$ in expressions like $(\mu+x)$, we derive the formula defining $\kappa$ via $\varepsilon$ and $\mu, \kappa \approx 1-(\varepsilon / 2 \mu)^{2}$, and get the cubic equation with respect to $\mu$ :

$$
\mu^{3}+c_{1} \mu^{2}+c_{2} \mu+c_{3}=0,
$$

where $\quad c_{1}=\sigma_{0}-1, \quad c_{2}=\varepsilon^{2} \sigma_{0} \cosh ^{2}\left[k_{u} y_{0}\right] / 4, \quad$ and $c_{3}=\varepsilon^{2}\left(2+\sigma_{0}\right) \cosh ^{2}\left[k_{u} y_{0}\right] / 8$. The discriminant analysis $D\left(\varepsilon, \sigma_{0}, y_{0}\right)=p^{3} / 27+q^{2} / 4 \quad\left(p=-c_{1}^{2} / 3+c_{2}, \quad q=\right.$ $\left.2 c_{1}^{3} / 3^{3}-c_{1} c_{2} / 3+c_{3}\right)$ of cubic Eq. (15) shows that $D(\varepsilon$, $\left.\sigma_{0}, y_{0}\right)<0$ in the region $\sigma_{0}<\sigma_{0}^{\text {crit1 }}$, and $D\left(\varepsilon, \sigma_{0}, y_{0}\right)>0$ in the region $\sigma_{0}>\sigma_{0}^{\text {crit1 }}$. The quantity $\sigma_{0}^{\text {crit1 }}\left(\varepsilon, y_{0}\right)$ is a solution to equation $D\left(\varepsilon, \sigma_{0}, y_{0}\right)=0$ and equals

$$
\begin{aligned}
\sigma_{0}^{\text {crit } 1}= & -\frac{1}{2}\left(\frac{9 \varepsilon \cosh \left[k_{u} y_{0}\right]}{2}\right)^{2 / 3} \\
& -\frac{3}{4}\left(\frac{\varepsilon \cosh \left[k_{u} y_{0}\right]}{6}\right)^{4 / 3}+\frac{7 \varepsilon^{2}}{18} .
\end{aligned}
$$

In the region $\sigma_{0}<\sigma_{0}^{\text {critl }}$, the solution to Eq. (15) has the following form:

$$
\mu=-\left(1-\sigma_{0}\right) / 3-2 \sqrt{-p / 3} \cos [(\alpha+2 \pi) / 3],
$$

where $\cos \alpha=-q /\left[2 \sqrt{-(p / 3)^{3}}\right]$. In the region $\sigma_{0} \geq$ $\sigma_{0}^{\text {crit } 1}$ the solution to Eq. (15) reads 
$\mu=-\frac{1-\sigma_{0}}{3}-\frac{p}{3(\sqrt{D}-q / 2)^{1 / 3}}+(\sqrt{D}-q / 2)^{1 / 3}$.

The case $\sigma_{0}<\varepsilon$ should be treated separately and we found that the trajectories remain unchanged, but to calculate the cyclotron frequency we have to use another formula $\Omega_{c}=\left\{\omega_{c}^{2}+\omega_{\beta}^{2} \cosh ^{2}\left[k_{u} y_{0}\right]\right\}^{1 / 2}$.

The dependence of normal frequencies $\Omega_{u}$ and $\Omega_{c}$ on normalized partial cyclotron frequency $\omega_{c} / \omega_{u}$ is demonstrated in Fig. 2 using the analytical expressions (11), (17), and (18) (solid lines) and the numerical simulation results of the equations generated by (9) (dots). We see that the normal frequencies substantially differ from their partial analogies near the magnetoresonance $\Omega_{c} \sim \Omega_{u}$. Note that in our analytical study we ignore the adiabatic undulator section and the interaction between different nonlinear internal resonances (B6). Nevertheless, as we see from Figs. 2-5 such an approximation gives a good agreement between the analytical and numerical results. In fact, the adiabatic undulator entrance "improves integrability" of (9) and weakens the interaction between the internal resonances (B6) so that we may treat different internal resonances separately while simultaneously neglecting the adiabatic undulator section.

It is well known that the electron dynamics is characterized by a negative-mass regime near the magnetoresonance [16]. The graphical illustration of this effect is presented in Fig. 3, where we plot average axial velocity $\bar{v}_{\|}$as a function of normalized energy $\mathcal{E} / m_{e} c^{2}$ using the formulas (17) and (18) and the results of the numerical simulations. It must be stressed that, in the self-consistent simulations presented in Sec. V, the initial beam energy, $\gamma_{0}$, is slightly higher than the energy, $\gamma^{\text {trans }}$, that corresponds to a transition between positive- and negative-mass regimes ( $\gamma^{\text {trans }}$ is a solution to the equation $d \bar{v}_{\|} / d \gamma_{0}=0$ ).

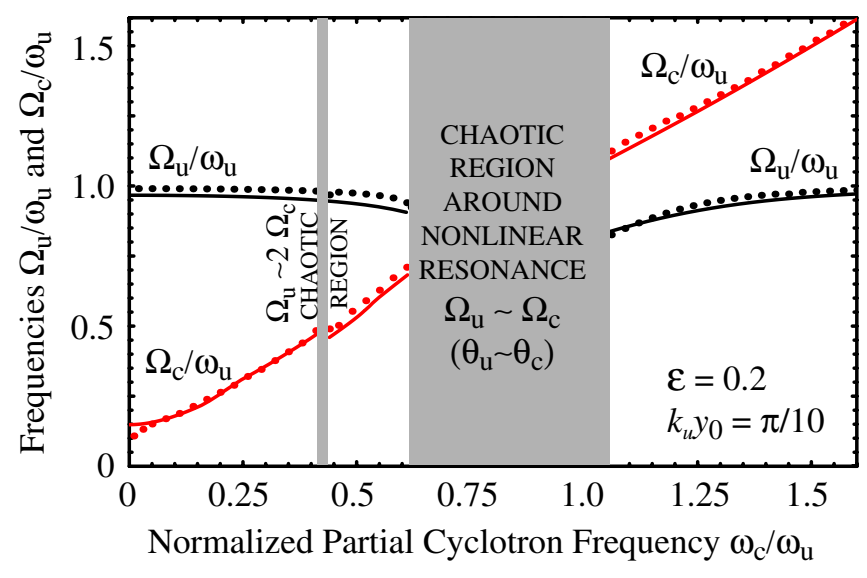

FIG. 2. The normal undulator and cyclotron frequencies vs the normalized partial cyclotron frequency. Solid lines are for the analytical results, while dots correspond to the results of the numerical simulation. In the numerical simulations the adiabatic undulator entrance is taken into account.

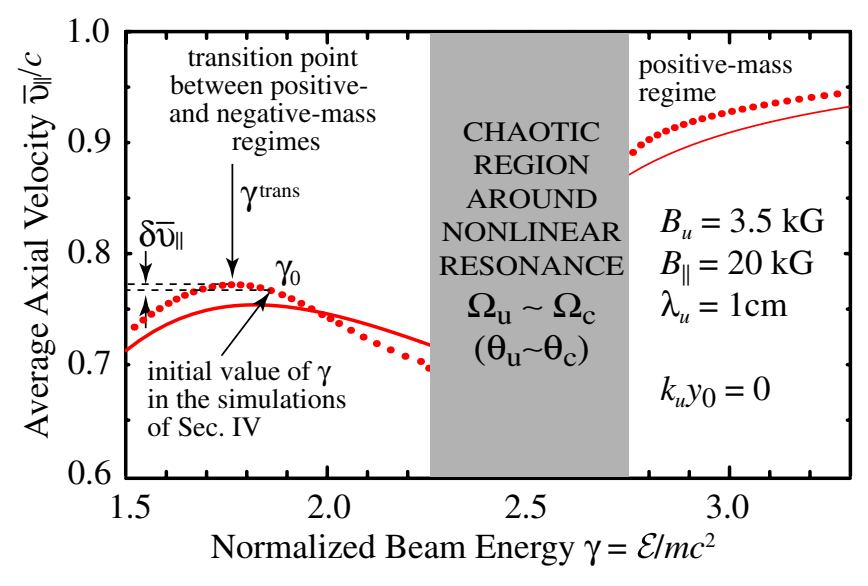

FIG. 3. Illustration of the negative-mass effect for a test electron in the pump field. The average axial velocity is a function of the beam energy. The curves are obtained using analytical formulas (17) and (18) while the dots represent the results of the numerical simulation.

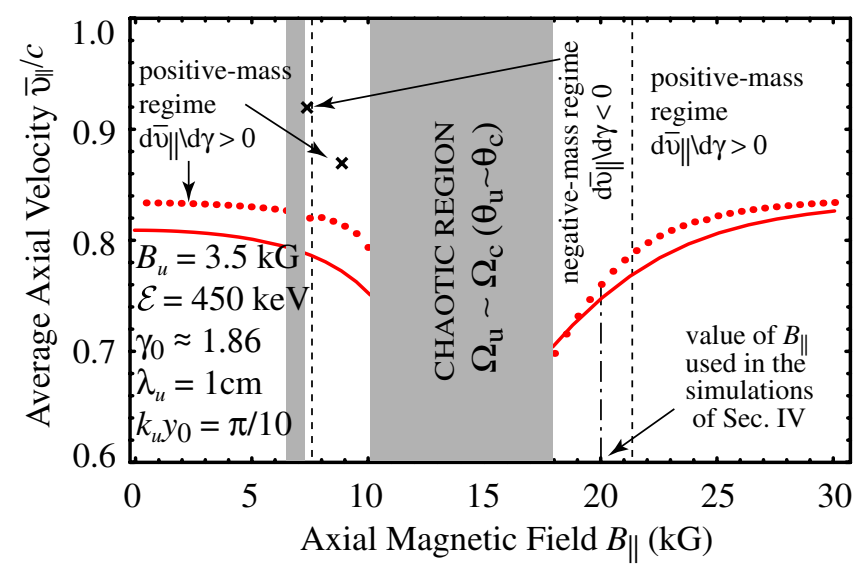

FIG. 4. The average axial velocity is a function of the axial magnetic field. The curves and the dots are for the analytical formulas (17) and (18) and the simulation results, respectively.

Because of such a choice electrons remain longer in synchronism with the wave because the beam energy variation causes the variation of the average axial velocity in the second order of smallness and the resonant condition $\omega=\left(k_{u}+k_{z}\right) \bar{v}_{\|}(\gamma)$ remains fulfilled for a wider range of the beam energy variation. For a fixed initial beam energy, the electron dynamics is also characterized by the positiveand negative-mass regimes depending on the value of axial magnetic field $B_{\|}$. This is shown in Fig. 4.

\section{B. Chaotic motion}

The equation set (B5) has a lot of nonlinear resonances (B6) $\left(n \vartheta_{u} \approx m \vartheta_{c}\right)$ between the undulator and cyclotron degrees of freedom, therefore one can expect appearance of chaotic dynamics in the system behavior. It was demonstrated in [15] that the main chaotic sea occupies a region of phase space with positive and negative axial momentum. 
That is, the axial velocity of a chaotic orbit can become negative even though the initial velocity is large and positive. Thus, the average axial velocity is equal to zero and one expects no beam transport. Indeed, the experiments $[18,19]$ in the beam transport in combined undulator and axial fields demonstrate that the beam transport vanishes if the FEL parameters correspond to the magnetoresonance regime. We found that the position and the boundaries of this transport gap are in an excellent agreement with the position and the boundaries of the chaotic regions. So, the electron chaotization is a strong harmful effect and the chaotic regions must be avoided in the experimental setups so that below we derive simple analytical formulas describing the chaotic region location and calculate the Lyapunov exponent map. The motion becomes stochastic if the difference between the undulator and cyclotron frequencies becomes less than the betatron frequency:

$$
\begin{aligned}
\left|\Omega_{u}-\Omega_{c}\right| & \leq \sqrt{2} \omega_{\beta} \cosh \left[k_{u} y_{0}\right] \\
(|\kappa-\sigma| & \left.\leq \varepsilon \cosh \left[k_{u} y_{0}\right]\right) .
\end{aligned}
$$

Such a criteria was initially proposed in [13] and proved numerically. With the derived $x$ and $\sigma$, we get the expression describing the location of the chaotic region:

$$
\begin{aligned}
& \sigma_{0}^{\text {crit } 1} \leq \sigma_{0} \leq \sigma_{0}^{\text {crit } 2}, \\
& \sigma_{0}^{\text {crit } 2}=\frac{2}{3}+\frac{28 \varepsilon \cosh \left[k_{u} y_{0}\right]}{27}+\frac{5 \varepsilon^{2} \cosh ^{2}\left[k_{u} y_{0}\right]}{18} .
\end{aligned}
$$

It turns out that the chaotization condition is inconsistent with the solution of equation set (12) for $\varepsilon$ less than the minimal value of $\varepsilon^{\mathrm{min}}$ :

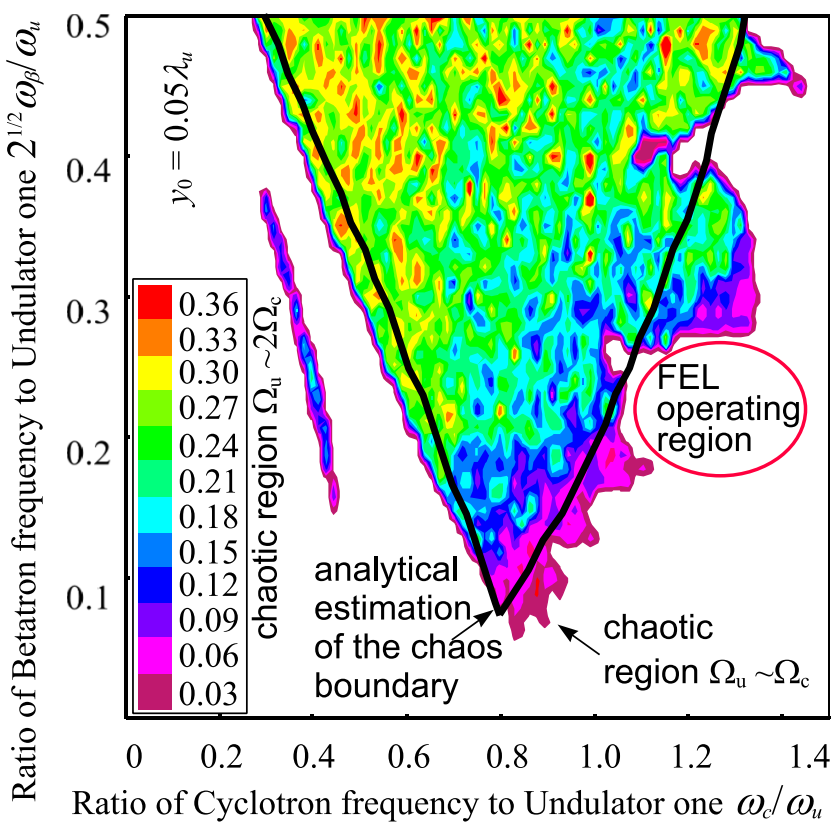

FIG. 5. The major Lyapunov exponent map. Black solid lines show the boundaries of the chaotic region according to the analytical formulas (20).

$$
\varepsilon^{\min } \cosh \left[k_{u} y_{0}\right]=0.0786 .
$$

This implies that there is no chaotic region for $\varepsilon<\varepsilon^{\mathrm{min}}$. In Fig. 5 we have illustrated the results of the numerical calculations for the major Lyapunov exponent. The solid lines calculated using Eq. (20) show the boundaries of the chaotic region. They are in good agreement with the results of the numerical simulation.

In what follows we analyze the studied FEL within the region of the regular dynamic.

\section{THE REDUCED MODEL OF THE FEL}

Using methods of nonlinear dynamics, one can construct a time-dependent reduced FEL model [20] that allows for the intricate dynamics of electrons in the pump magnetic field (1), the effect of the electron beam finite-thickness, and the intramode scattering in the profiled waveguide (the intramode scattering acts actually as a feedback). The reduced FEL model reads

$$
\begin{gathered}
\frac{\partial^{2} \psi}{\partial \zeta^{2}}=-Y\left(y_{0}\right) Z(\zeta) \operatorname{Re}\left\{\left(F_{+}+F_{-}\right) e^{i \psi}\right\}, \\
\frac{\partial F_{+}}{\partial \zeta}-i \delta_{z}(\zeta) F_{+}+\left(\frac{\bar{v}_{\|}}{v_{g r}}-1\right) \frac{\partial F_{+}}{\partial \tau}=J^{e}(\zeta, \tau), \\
\frac{\partial F_{-}}{\partial \zeta}+i\left(2 k_{z} \ell_{g}-\delta_{z}\right) F_{-}-\left(1+\frac{\bar{v}_{\|}}{v_{g r}}\right) \frac{\partial F_{-}}{\partial \tau}=\frac{\partial k_{z}}{\partial \zeta} \frac{F_{+}}{2 k_{z}}, \\
J^{e}(\zeta, \tau)=\frac{Z(\zeta)}{\pi Y_{b}} \int_{-Y_{b} / 2}^{Y_{b} / 2} d y_{0}^{\prime} \int_{-\pi}^{\pi} d \psi_{0}^{\prime} Y\left(y_{0}^{\prime}\right) e^{-i \psi\left(\zeta, \tau ; y_{0}^{\prime}, \psi_{0}^{\prime}\right),} \\
\left.\psi\right|_{\zeta=0}=\psi_{0},\left.\quad \frac{\partial \psi}{\partial \zeta}\right|_{\zeta=0} \equiv \delta_{y}\left(y_{0}\right)=\frac{\ell_{g} \omega}{\bar{v}_{\|}(0)}-\frac{\ell_{g} \omega}{\bar{v}_{\|}\left(y_{0}\right)}, \\
\left.F_{+}\right|_{\zeta=0}=F_{0},\left.\quad F_{-}\right|_{\zeta=L / \ell_{g}}=0 .
\end{gathered}
$$

Here $\zeta=z / \ell_{g}$ and $\tau=\left(\bar{v}_{\|} t-z\right) / \ell_{g}$ are the dimensionless longitudinal coordinate and "retarded time" [21];

$$
F_{ \pm}=-\frac{i e c \omega^{2} \ell_{g}^{2}}{\sqrt{2} \bar{v}_{\|}^{3} \bar{\gamma}_{\|}^{2} \mathcal{E}} \frac{\omega_{\beta}}{\Omega_{u}} V_{ \pm} e^{i \int_{0}^{\zeta} \delta_{z}\left(\xi^{\prime}\right) d \zeta^{\prime}}
$$

is the normalized field amplitude; $\psi\left(z, t ; y_{0}, \psi_{0}\right)=k_{u} z+$ $\int_{0}^{z} k_{z}\left(z^{\prime}\right) d z^{\prime}-\omega t-\ell_{g}^{-1} \int_{0}^{z} \delta_{z}\left(z^{\prime}\right) d z^{\prime}$ is the ponderomotive phase; $\psi_{0}$ and $y_{0}$ are the initial entrance phase and the initial transverse displacement of the electron position from the undulator symmetry plane $y=0$;

$$
\ell_{g}^{-3}=\frac{1}{\bar{\beta}_{\|}^{3} \bar{\gamma}_{\|}^{3}} \frac{I_{0}}{I_{\alpha}} \frac{2 \pi \omega^{2}}{k_{z}^{0} S c^{2}} \frac{\omega_{\beta}^{2}}{\Omega_{u}^{2}},
$$

where $I_{\alpha}=m_{e} c^{3} / e \approx-17 \mathrm{kA}$ is the Alfvén current (recall that $\left.e, I_{0}<0\right)$. The parameter $\ell_{g}$ is called the gain length [21] (the spatial growth rate of the FEL without the 
axial magnetic field is equal to $\ell_{g}^{-1}$ for zero detuning). The explicit dependence of the reduced FEL model on the transverse electron position and the axial position are given by the relations

$$
Y=\frac{\Omega_{u}^{2}\left(y_{0}\right) \cosh \left[k_{u} y_{0}\right]}{\Omega_{u}^{2}\left(y_{0}\right)-\Omega_{c}^{2}\left(y_{0}\right)} \cos \left[\frac{\pi y_{0}}{w(z)}\right], \quad Z=\sqrt{\frac{k_{z}^{0} b}{k_{z}(z) w(z)}}
$$

The dimensionless longitudinal detuning parameter is

$$
\delta_{z}(\zeta)=\ell_{g}\left(k_{z}(\zeta)+k_{u}-\frac{\omega}{\bar{v}_{\|}(0)}\right)
$$

Our model (22) is exactly coincident with the ColsonBonifacio model [22,23] if a free-space case is employed, the axial magnetic field equals zero, and the electron beam is ideally thin and ultrarelativistic.

It turns out that the model (22) depends solely on the average axial velocity via the detuning parameters, but it is independent of the particular scalar components of the initial velocity. This results in that the FEL efficiency is only dictated by average axial velocity spread $\delta \bar{v}_{\|}\left(t_{e}\right)$ that can be written as $\delta \bar{v}_{\|} \propto \delta v_{z}+\varepsilon \delta v_{x}+\varepsilon^{2} \delta v_{y}$, where $\delta v_{i}\left(t_{e}\right)(i=x, y, z)$ is the magnitude of the initial velocity spread $\left(\delta v_{i} / \bar{v}_{\|} \ll 1\right)$. Recall that $\varepsilon=\sqrt{2} \omega_{\beta} / \omega_{u}$ is a small parameter so that one first needs to minimize the initial axial velocity spread. The velocity spread changes the efficiency insignificantly if the detuning caused by the spread is much smaller than unity [21]. For the ideally thin beam $\left(Y_{b} \rightarrow 0\right)$ it yields the condition

$$
\frac{\left(k_{z}^{0}+k_{u}\right) \ell_{g}}{Y^{2 / 3}} \frac{\mu_{\bar{v}_{\|}}}{\bar{v}_{\|}} \ll 1,
$$

where $\mu_{\bar{v}_{\|}}$is the variance of $\delta \bar{v}_{\|}$. It was shown in [24] (see also the results of the numerical simulation in [13]) that essential decreases in the sensitivity of the efficiency to the initial beam spread can be obtained if the undulator frequency is close to the cyclotron one (multiplier $Y^{-2 / 3}$ attains its minimal value).

In general, the ponderomotive potential enhances as the undulator frequency tends to the cyclotron one $\left[Y\left(y_{0}\right)\right.$ increases]. This means a stronger coupling between the wave and electrons. Such an effect referred to as the magnetoresonance is well known in the literature [17] and the recent detailed study [13] confirmed the usefulness of such a regime for a planar FEL configuration. However, the magnetoresonance effect is not so effective when the beam has a finite thickness. Electrons with the different initial transverse positions, $y_{0}$, undergo the action of the different magnitudes of the pump magnetic field (1). Therefore, electron average velocity $\bar{v}_{\|}$depends on $y_{0}$ and this dependence particularly strong near the magnetoresonance. At the same time $\bar{v}_{\|}$governs the initial

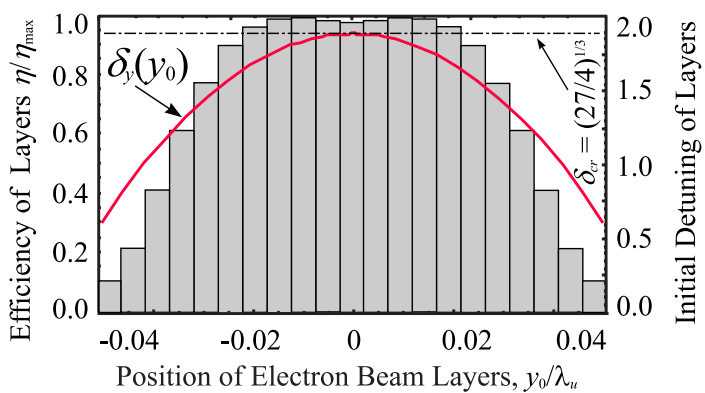

FIG. 6. The relative efficiency (histogram) and the initial detuning (red solid line) of electron beam layers vs the normalized transverse displacement of layers from the symmetry plane $y=0$.

"transverse" detuning $\delta_{y}\left(y_{0}\right)$ between the electron and the wave. Hence, the value of $\delta_{y}\left(y_{0}\right)$ changes across the beam, and the contribution of different electrons to the total efficiency might be rather different. To demonstrate this effect we simulate Eqs. (22) for the parameters close to the experiment [25] and assume there is additional axial magnetic field $20 \mathrm{kG}$ as well. In the simulation we split the beam into 21 layers in the transverse cross section. Each layer is also uniformly distributed into 50 macroparticles entering within one wave period. Recall that the physical system under study is homogenous in the $x$ direction. The results are shown in Fig. 6. The internal layer operates in the regime of optimal (with respect to efficiency) detuning $\delta_{z}(\zeta)=\delta_{c r}=(27 / 4)^{1 / 3}$ and $\delta_{y} \equiv 0$, but the external layers operate with the nonoptimal detuning because of the variation in $\delta_{y}$ (illustrated in Fig. 6 as the solid line) across the beam. Let us remind that according to the onedimensional Colson-Bonifacio model the efficiency depends only on detuning parameter $\delta$ and attains its maximal value if $\delta$ is slightly smaller than the critical value $\delta_{c r}=(27 / 4)^{1 / 3}$ (see Fig. 2 in $[21,26]$ ).

Another important feature of the model (22) is that it takes into account the effect of waveguide profiling. This effect exhibits the coupling between the forward and backward waves because of the intramode scattering. Wave number $k_{z}$ depends on axial position $z$ through varying waveguide width $w(z)$ and, as a result, "longitudinal" detuning $\delta_{z}$ is also a function of $z$ and its control can be used to govern the beam-wave interaction.

\section{CONTROL OF THE BEAM-WAVE INTERACTION: FEL WITH THE OPTIMIZED WAVEGUIDE PROFILE}

Now I discuss the physical principle of the control of the beam-wave interaction. For simplicity we consider the steady-state regime and the thin beam. We also neglect the backward wave generation assuming that $w(z)$ is a slow function of $z$. Rewriting the complex amplitudes of the wave and ponderomotive current as $F_{+}=\left|F_{+}\right| e^{i \alpha}$ and $J^{e}=\left|J^{e}\right| e^{i u_{*}}$ and using Eqs. (22) we arrive at the system: 


$$
\begin{aligned}
\frac{d^{2} \psi}{d \zeta^{2}} & =-\left|F_{+}\right| \cos (\alpha+\psi), \\
\frac{d\left|F_{+}\right|}{d \zeta} & =\left|J^{e}\right| \cos \left(\alpha-u_{*}\right), \\
\frac{d \alpha}{d \zeta} & =\delta_{z}(\zeta)-\left|J^{e}\right| \sin \left(\alpha-u_{*}\right) /\left|F_{+}\right| . \\
u_{*} & =\operatorname{Arg}\left(\frac{1}{\pi} \int_{0}^{2 \pi} e^{-i \psi} d \psi_{0}\right), \\
\left|J^{e}\right| & =\frac{1}{\pi}\left|\int_{0}^{2 \pi} e^{-i \psi} d \psi_{0}\right| .
\end{aligned}
$$

Here $\psi,\left|F_{+}\right|$, and $\alpha$ are the unknown quantities governed by the differential equations, and $u_{*}$ and $\left|J^{e}\right|$ are given by definition. The phase of the current $u_{*}$ defines the position of the bunch center in the system of coordinates moving with the velocity of the beam ([4], p. 160; see also [27], p. 325). One can see that if the phase shift between the current and the wave, $\phi=\alpha-u_{*}$, belongs to the interval from $-\pi / 2$ to $\pi / 2$, then the right-hand side of the equation governing wave amplitude $\left|F_{+}\right|$[second equation in the upper line of (27)] has a positive sign and the amplitude itself grows. The phase shift $\phi$ governs the energy transfer from the beam to the wave because the local interaction power is $d P / d \zeta \propto\left|J^{e}\right|\left|F_{+}\right| \cos \phi$. This implies that we can increase the efficiency by controlling $\phi$ along the interaction region by changing the detuning parameter $\delta_{z}(\zeta)$ in an appropriate way. The idea of such an optimization was originally proposed in TWT theory [28]. Here, for example, we demonstrate the simple indirect optimization method [28]. Now assume that the phase shift, $\phi$, satisfies the relation:

$$
\begin{aligned}
& \alpha-u_{*} \equiv \phi_{\mathrm{opt}}(\zeta)=-\pi \sin ^{2} \frac{\pi \zeta}{2 L_{\mathrm{pr}}}, \quad \zeta \leq L_{\mathrm{pr}}, \\
& \alpha-u_{*} \equiv \phi_{\mathrm{opt}}(\zeta)=0, \quad \zeta>L_{\mathrm{pr}},
\end{aligned}
$$

where $L_{\mathrm{pr}}$ is the start point of the region with the permanent value of $\phi$. We have to find $\psi$ and $\left|F_{+}\right|$using Eqs. (27) and (28) [in the right-hand sides of Eqs. (27) the expression $\left(\alpha-u_{*}\right)$ should be replaced by $\left.\phi_{\text {opt }}\right]$. Then we can restore the information about the waveguide profile using the equation for the detuning parameter that follows from (27):

$$
\delta_{z}(\zeta)=\frac{d u_{*}}{d \zeta}+\frac{d \phi_{\mathrm{opt}}}{d \zeta}+\left(\left|J^{e}\right| \sin \phi_{\mathrm{opt}}\right) /\left|F_{+}\right|
$$

The simulation results of the amplitudes of the wave $\left|F_{+}\right|$ and the current $\left|J^{e}\right|$ and the phase shift $\phi$ are shown in Fig. 7. In this figure we also plotted the FEL characteristics for the constant detuning. We can see that the wave amplitude can be significantly enhanced (efficiency increased several times). However, the demonstrated optimization technique is useful only for a slightly improved efficiency because the waveguide profiles are to be rather complicated

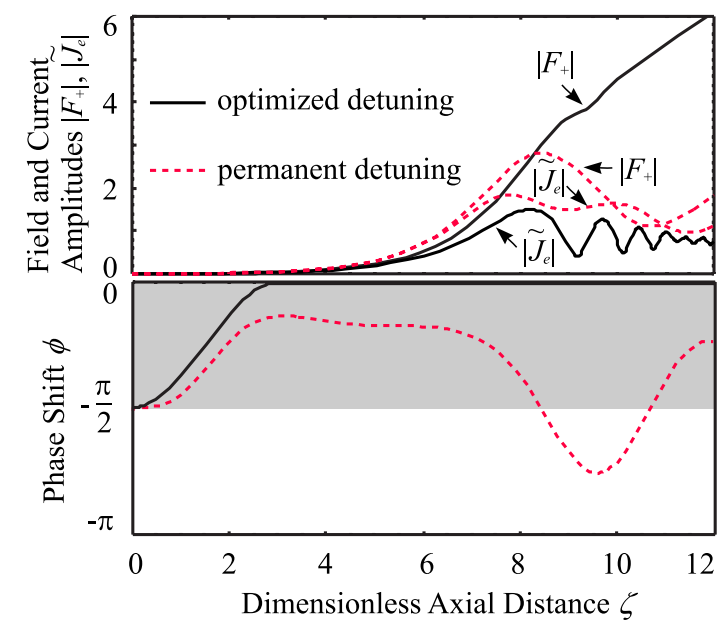

FIG. 7. The simulation results for the FEL with and without the phase shift optimization are demonstrated.

from the practical point of view in an effort to considerably increase the efficiency. Then more elaborated mathematical approaches, which simultaneously allow one to control the practical realizability of optimal waveguide profiles, should be used. In this paper we apply some type of a genetic algorithm [29] to perform the FEL optimization. The principle of evolutionary optimization is rather simple: we generate a lot of waveguide profiles and then perform numerical simulation of the reduced model (22) using these profiles. Then we choose the best profiles, cross and modify them, and perform the simulation again. As a result, one can find a few best profiles. Finally, we must check that these found optimal profiles are really useful. To this end we have to simulate the nonsimplified original model (formulated in Sec. II) using these profiles. The mathematical formulation of the nonsimplified FEL model is very close to that of the code MEDUSA [16], so similar numerical methods are used. In particular, the fourth-order Adams-Moulton predictorcorrector scheme is used in order to integrate the equations of motion. The implicit and absolutely stable scheme no. 3 of Table 8.1 in [30] is used to solve the partial differential diffusionlike equation for the wave amplitude. The accuracy of this simple scheme is $O(\Delta t)+O\left[(\Delta z)^{2}\right]$, where $\Delta t$ and $\Delta z$ are the steps in time and axial direction, respectively. Typically, $\Delta z \sim \lambda / 10 \sim \lambda_{u} / 20$ and $\Delta t \sim 2 \pi / \omega$. In the right-hand side of Eq. (3) the integral with respect to the initial phase is calculated by using the rectangle rule. Since the integrand is a periodic function with respect to the initial phase and the integration is performed over the period, this elementary method provides an adequate accuracy. To perform integration with respect to the initial transverse positions of electrons, one employs the trapezoidal rule. The accuracy of the self-consistent simulations is controlled by checking the energy conservation law. Typical relative error is around $10^{-5}-10^{-6}$. The simulation code was verified by comparing its results with the analytical ones [13] and by comparing with the numerical data given in $[11,31]$ 


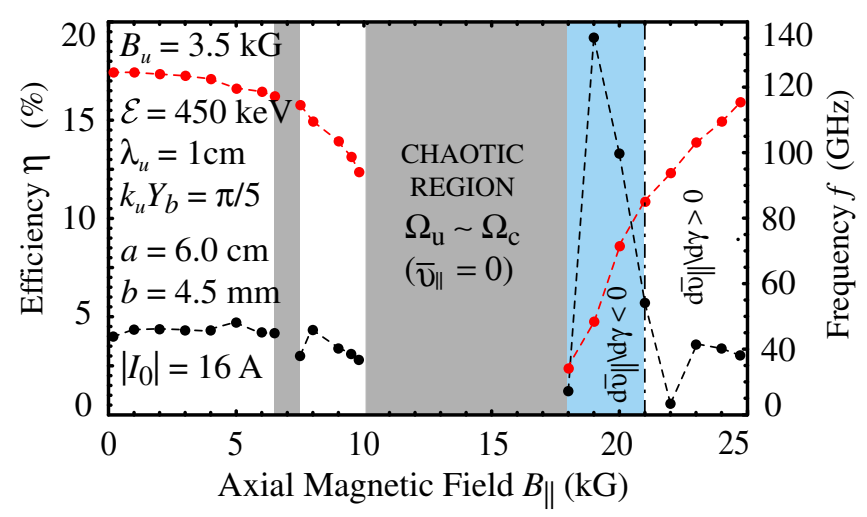

FIG. 8. The FEL efficiency and the resonant frequency vs the axial magnetic field. The results of the simulation are based on the nonsimplified model.

and [11] (the pump magnetic field in this case was modified in an appropriate way). The varying waveguide profile can be represented as

$$
w(z)=b(1+A(z) \sin [\nu(z)+\varphi(z)])
$$

where

$$
\begin{aligned}
& A(z)=\sum_{n=1}^{N} A_{n}(z / C)^{n}, \quad \nu(z)=\sum_{n=1}^{N} \nu_{n}(z / C)^{n}, \\
& \varphi(z)=\varphi_{0}\left(\sin ^{2}\left[\pi z / 2 \lambda_{u}\right] U\left[\lambda_{u}-z\right]+U\left[z-\lambda_{u}\right]\right) .
\end{aligned}
$$

Here $U[z]$ is the Heaviside function and $A_{n}, \nu_{n}, C$, and $\varphi_{0}$ are the adjusting constants. Let us consider the results of the optimization for a practical example.

The FEL parameters are chosen to be close to the parameters of experiment [25]: $450-\mathrm{kV}$ beam voltage,
$\left|I_{0}\right|=16$-A beam current, $1.0 \mathrm{~mm} \times 2.0 \mathrm{~cm}$ sheet electron beam interacts with the $\mathrm{TE}_{01}$ mode (the field varying along the narrow wall) of the $4.5 \mathrm{~mm} \times 6.0 \mathrm{~cm}$ rectangular waveguide. The undulator magnitude increases adiabatically within six periods and the undulator is characterized by parameters $B_{u}=3.5 \mathrm{kG}$ and $\lambda_{u}=1.0 \mathrm{~cm}$ in the regular region. A $1.5-\mathrm{kW}$ input signal with the $4.2 \mathrm{~mm}$ wavelength is injected. According to the linear FEL theory, the spatial growth rate attains its maximal value in the zone of regular beam dynamics slightly above the magnetoresonant value of the axial magnetic field. However, the nonlinear analysis strongly indicates, see Fig. 3, 4, and 8, the FEL efficiency is maximal if the axial magnetic field is chosen in such a way that the transition from the negativemass to positive-mass regimes occurs during the beamwave interaction. In that case electrons remain longer in synchronism with the wave at the nonlinear stage because the beam energy variation causes small variations of the average axial velocity, see Fig. 3, and the resonant condition remains fulfilled for a wider range of the beam energy variation. The maximal efficiency, $19 \%$, is achieved at $B_{\|}=19 \mathrm{kG}$ and the resonant frequency $48.4 \mathrm{GHz}$. Since we are interested in the $75 \mathrm{GHz}$ FEL amplifier, we will choose in further simulations the axial field being equal to $20 \mathrm{kG}$.

In Fig. 9(a) the results for the FEL with the axial field but without optimization are shown. Using the magnetoresonance effect, we can significantly enhance the efficiency. It was $4 \%$ efficiency without the axial field in the experiment [25] and it is $12 \%$ efficiency with the axial magnetic field. However, there is a weak interaction between the external beam layers and the microwave because different layers of the electron beam have different transverse detuning with
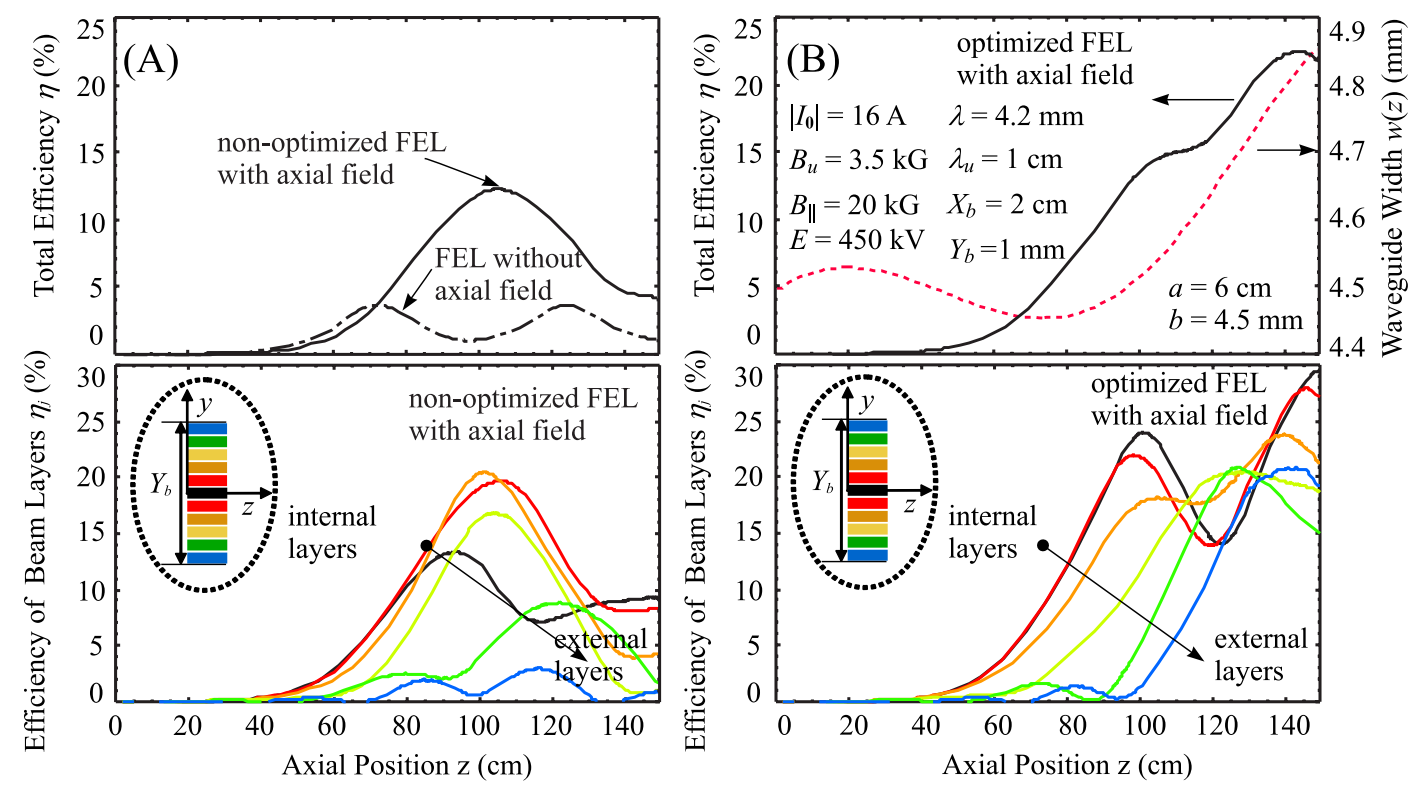

FIG. 9. The total FEL efficiency, the efficiency of electron beam layers, and the waveguide width vs the interaction length. The results of the simulation based on the nonsimplified model given in Sec. II are demonstrated. 
the wave due to the transverse inhomogeneity of the pump magnetic field. Geometric positions of different beam layers at the beginning of the interaction region are shown inside the dotted ellipse. The black curve is for the central layer. Other layers are displaced with respect to the symmetry plane $y=0$.

In Fig. 9(b) the results for the optimized FEL with the axial field are presented. The waveguide profile is characterized by the parameters [see Eq. (30)]: $N=3$, $A_{1}=0.223, A_{2}=-0.894, A_{3}=-0.964, \nu_{1}=-1.902$, $\nu_{2}=0.293, \nu_{3}=0.901, \varphi_{0}=0.723$, and $C=200$. The maximal relative deviation of the waveguide width is $8 \%$ and the maximal and minimal widths are around 4.9 and $4.4 \mathrm{~mm}$, respectively. The dimensionless slope of the waveguide taper $\epsilon_{w}=k_{u}^{-1} \partial_{z} \ln w \approx 0.0002$ is much smaller than the normalized growth rate of the wave $\operatorname{Im} \delta k_{z} / k_{u} \approx 0.0029$. Using the waveguide with the optimized profile, one can double the efficiency so that the final efficiency is around $22 \%$. We also see that the external layers interact with the wave much more effectively in the optimized FEL. So, by changing the waveguide profile we control beam-wave interaction thus increasing the FEL efficiency.

The distribution of the microwave power along the interaction region for different moments of time is presented in Fig. 10. We checked that the proposed FEL amplifier reaches a steady-state regime and it takes around 8 ns. The field transition time $t_{\text {tr }}=L / \bar{v}_{\|}+\left(\bar{v}_{\|}^{-1}-\right.$ $\left.v_{\mathrm{gr}}^{-1}\right) l_{u} \tau_{\text {tr }}$, where $\tau_{\text {tr }}$ is the dimensionless transition time and its value is typically 150-200, is comparable with the electron transit time $\tilde{t}=L / \bar{v}_{\|} \approx 6.52 \mathrm{~ns}$.

It should be mentioned that the applied axial magnetic field $\vec{B}_{\|}$not only allows one to increase the FEL efficiency

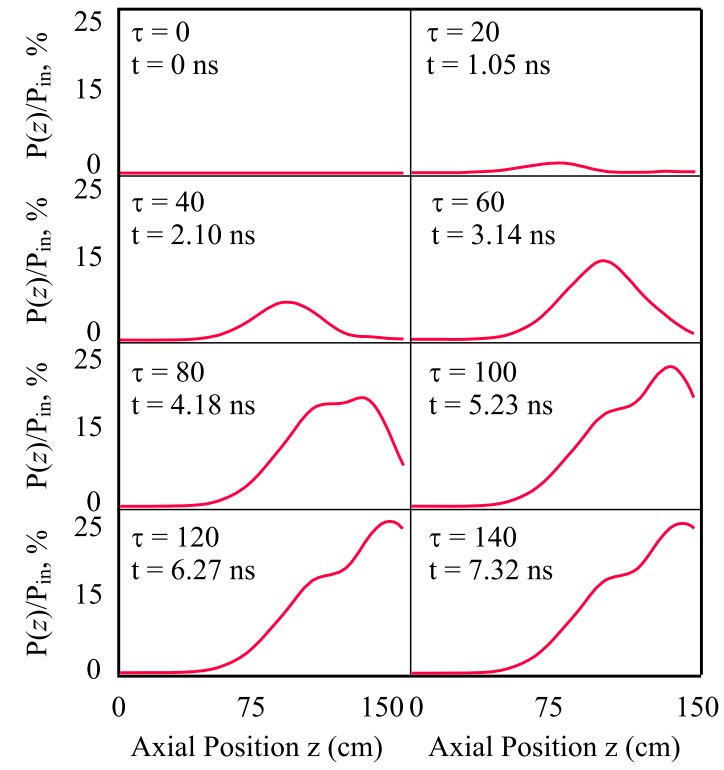

FIG. 10. The normalized power vs the interaction length at different moments of time. substantially, but also causes a beam divergence due to the electron drift $\vec{B}_{\|} \times \nabla \vec{B}_{u}\left(\vec{B}_{u}\right.$ is the undulator field) in the $x$ direction. However, ponderomotive potential $W$ is independent of $x$ coordinates of electrons because the microwave and pump magnetic field are homogeneous in the $x$ direction. As a result, the beam-wave interaction is not drift dependent unless electrons fall down on the waveguide walls. It is checked that electrons do no touch the walls in the situations of interest (see Fig. 11). Since the drift velocity depends on $y_{0}$ as $\sinh \left[2 k_{u} y_{0}\right]$, the central layer $y_{0}=0$ does not undergo the drift effect, whereas the beam layers displaced with respect to the symmetry plane $y=0$ drift toward walls with different velocities.

Depending on specific parameters, a number of parasitic modes, including a mixture of the $\mathrm{TE}_{21}$ and $\mathrm{TM}_{21}$ modes, may be excited by the beam. However, for the given above FEL configuration parasitic modes affect weakly the interaction of the beam with the dominant $\mathrm{TE}_{01}$ mode due to several reasons. Coupling between the wave and the beam strongly depends on the electric field distribution in the transverse plane and the beam location. From Fig. 12 we see that the beam position coincides with the field maximum for the $\mathrm{TE}_{01}$ mode and the field distribution is homogeneous in the $x$ direction, therefore the beam-wave coupling is high. But the contributions of different parts of the beam annihilate each other for $\mathrm{TE}_{11}$ and $\mathrm{TM}_{11}$ because the electric field $E_{x}\left(E_{y}\right)$ has different signs
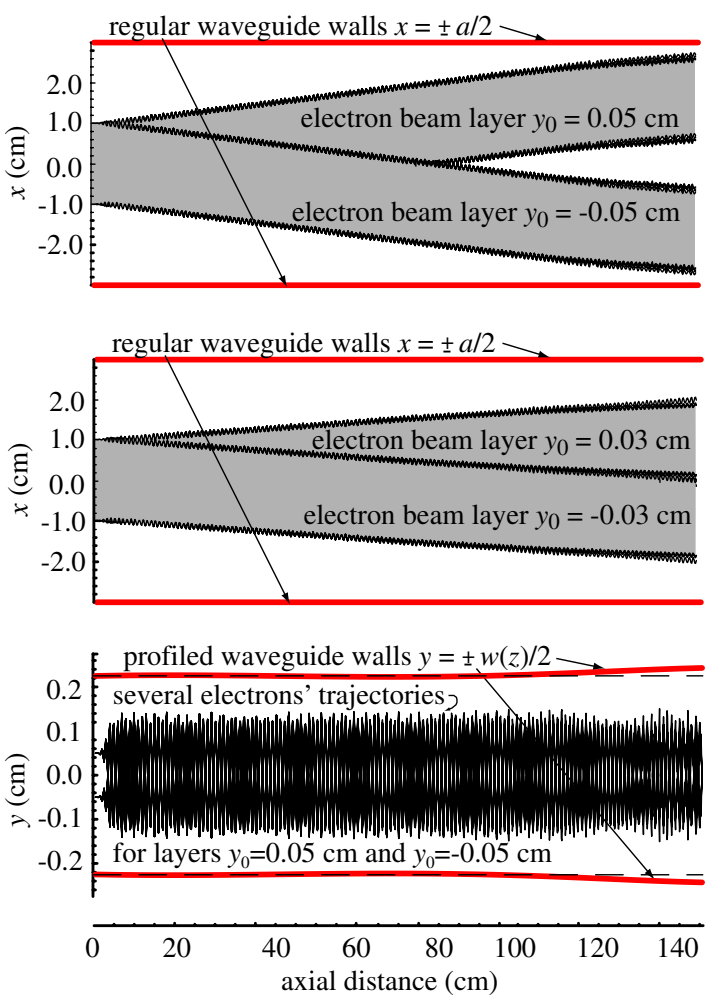

FIG. 11. Some electrons' trajectories in the $x-z$ and $y-z$ planes are shown. 

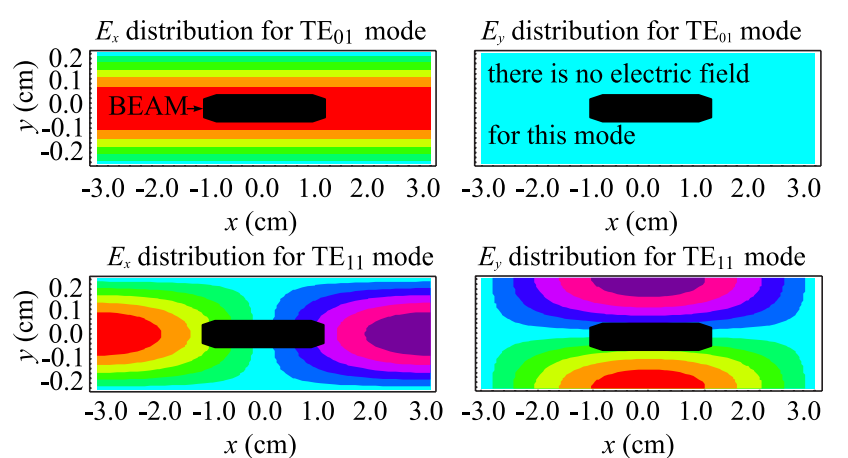

$E_{y}$ distribution for $\mathrm{TE}_{11}$ mode

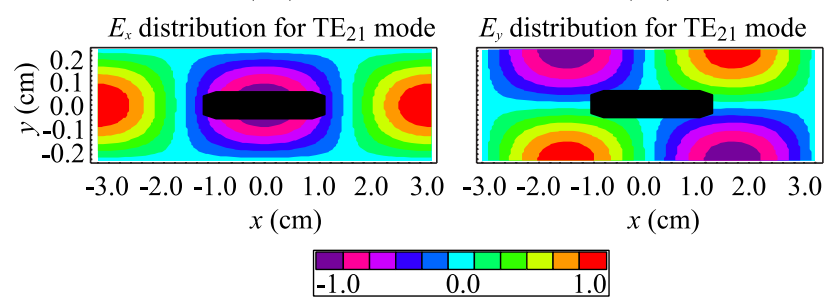

FIG. 12. The field distributions of $\mathrm{TE}_{01}, \mathrm{TE}_{11}$, and $\mathrm{TE}_{11}$ modes and the beam initial position are demonstrated.

for $x>0(y>0)$ and $x<0(y<0)$. As for the $\mathrm{TE}_{21}$ and $\mathrm{TM}_{21}$ modes, these modes are quite effectively matched to the beam via $E_{x}$, but their resonant frequency is $68.5 \mathrm{GHz}$ whereas the FEL is seed at the frequency $71.4 \mathrm{GHz}$ and the bunching occurs at a higher frequency than it is necessary for the excitation of $\mathrm{TE}_{21}$ and $\mathrm{TM}_{21}$ modes. In the experiment [8] the output power from the tapered wiggler was nearly all $(\sim 90 \%)$ in the fundamental mode whereas the resonant frequencies for the $\mathrm{TE}_{01}$ and $\mathrm{TE}_{21}$ modes were 34.6 and $32.5 \mathrm{GHz}$, respectively. Moreover, it was not reported about the parasitic modes excitation in experiment [25]. Besides, the beam drift leads to suppression of the $\mathrm{TE}_{21}-\mathrm{TM}_{21}$ modes excitation because, as we see from Figs. 11 and 12, at the end of the interaction region the phase shift between the external beam layers $y_{0}= \pm 0.05 \mathrm{~mm}$ and the central layer $y_{0}=0$ is around $\pi$. Therefore, the contribution of the different layers is partly annihilated and we may ignore parasitic modes.

\section{SUMMARY AND DISCUSSION}

The operation of a planar FEL amplifier with an axial magnetic field and an irregular waveguide is studied. The self-consistent model, which includes the excitation equation and the equations of motion along with the expressions for the radiated field and the microscopic current density, is formulated. In order to find the parameters and the waveguide profile that provide the maximal efficiency, one has to apply an optimization technique. However, the direct numerical optimization based on the nonaveraged FEL model fails to work because a vast amount of computational resources is required. In this paper, I propose another approach to the problem. The investigation is divided into several stages: initially the equations of motion and the excitation equation are partly integrated in an analytical way using methods of nonlinear dynamics. As a result, the reduced FEL model is derived in a special phase space. Then with this model and some principles of evolutionary computations (genetic algorithms) the numerical optimization of the waveguide profile is performed. Finally, the simulation of the nonsimplified original model using the found optimal waveguide profiles is carried out. So, one can come closer to understanding what increase in the efficiency can be achieved in practice.

There is no reversed-guide-field regime for a planar FEL configuration so that in the paper the magnetoresonance effect is employed to enhance the beam-wave interaction. According to the linear theory $[13,16,17]$, the spatial growth rate attains its maximal value in the zone of regular beam dynamics slightly above the magnetoresonant value of the guide magnetic field. However, the nonlinear analysis strongly indicates that the FEL efficiency is maximal if the axial magnetic field is about $20 \%-30 \%$ higher than its magnetoresonance value so that the transition from the negative-mass to positive-mass regimes occurs during the beam-wave interaction, see Figs. 3, 4, and 8. In that case electrons remain longer in synchronism with the wave at the nonlinear stage because the beam energy variation causes the variation of the average axial velocity only in the second order of smallness (at the transition point $d \bar{v}_{\|} / d \gamma=0$ ) and the resonant condition remains fulfilled for a wider range of the beam energy variation. Typically, the magnetoresonance allows one to increase the FEL efficiency by a factor of 5 or 6 if the beam is very thin and by a factor of 3 or 4 if the beam thickness is relevant. At the same time the magnetoresonance effect appears not only as an advantage, but the beam degradation and divergence caused by the electron dynamics chaotization occur $[15,18,19]$ if $\Omega_{u}$ is too close to $\Omega_{c}$. The simple analytical formulas describing the chaotic region location are derived in the paper so that our formulas (20) allow one to easily estimate the parameter region that has to be avoided in experimental setups. The applied axial magnetic field also causes the electron drift $\vec{B}_{\|} \times \nabla \vec{B}_{u}$ in the $x$ direction. Because of the drift the distance between narrow waveguide walls has to be larger than $X_{b}+2 x\left(L / v_{\|}\right)$, where $X_{b}$ and $L$ are the beam width and the interaction length, and the electron position $x(t)$ is given by (13). In the case of a wide sheet beam the waveguide width is mainly determined by the beam width, but the drift also has to be taken into account, see Fig. 11.

There are two types of detuning in the reduce model (22): "axial" detuning parameter that changes along the interaction region via the profiled waveguide width and the transverse detuning parameter that changes across the beam because the pump magnetic field is inhomogeneous and the average velocity of the electron depends on its initial transverse position. The transverse detuning causes layering of the thick beam and the degradation of the 
external layers' contribution into the total efficiency. The present paper demonstrates that the beam layering and the saturation effect can be overcome by the control of the beam-wave interaction. The physical mechanism of such a control is that by changing the waveguide profile one controls the axial detuning and thus regulates the phase shift between the ponderomotive wave and current. This phase shift defines the transfer of the energy between the beam and the wave and its regulation allows one to optimize the interaction.

The practical example of optimization of the FEL, whose parameters are close to those of the experiment [25], is demonstrated. The simulation results based on the nonsimplified model (see Sec. II) strongly indicate that combining the magnetoresonance effect with the optimized profile waveguide one can enhance the FEL efficiency by a factor of 5 or 6 . The efficiency in the experiment [25] was around 4\%. Applying the axial magnetic field, the efficiency has been increased up to nearly $12 \%$, but about $30 \%$ of electrons do not interact with the wave because of the initial transverse detuning. Following the waveguide optimization the efficiency has reached $22 \%$, in particular, due to a much more effective interaction between the external beam layers and the wave.

\section{ACKNOWLEDGMENTS}

I would like to acknowledge fruitful conversations with Dr. K. Ilyenko, Dr. A. Opanasenko, and Dr. O. V. Usatenko. Also, I would like to thank Dr. K. Ilyenko for drawing my attention to the work of Dr. Yu. V. Tarsov, with whom I am grateful for useful discussions as well. I am also grateful to referees, whose considered criticism allowed me to improve the exposition substantially. In addition, I would like to thank referee A for drawing my attention to the relevant impact of a transition between negative and positive mass regimes on the FEL efficiency.

\section{APPENDIX A: TIME-DEPENDENT EXCITATION OF AN IRREGULAR WAVEGUIDE}

The evolution of the resonant (synchronous) $\mathrm{TE}_{01}$ mode is governed by the $x$ component of the vector-potential $A_{x}^{s}$, which satisfies the wave equation

$$
\left(\nabla^{2}-\frac{1}{c^{2}} \frac{\partial^{2}}{\partial t^{2}}\right) A_{x}=-\frac{4 \pi}{c} j_{x}
$$

We seek a solution to the equation of the form

$A_{x}(\vec{r}, t)=\operatorname{Re} \int_{0}^{\infty} \bar{V}\left(z, \omega^{\prime}\right) \sqrt{\frac{b}{w(z)}} \cos \left(\frac{\pi y}{w(z)}\right) e^{-i \omega^{\prime} t} d \omega^{\prime}$.

Substituting (A2) into (A1) we derive the excitation equation for the Fourier amplitude $\bar{V}\left(z, \omega^{\prime}\right)$ :

$$
\begin{aligned}
& \left\{\frac{\partial^{2}}{\partial z^{2}}+k_{z}^{2}\left(z, \omega^{\prime}\right)\right\} \bar{V}\left(z, \omega^{\prime}\right) \\
& =-\frac{8 \pi}{S c} \sqrt{\frac{b}{w(z)}} \int_{-a / 2}^{a / 2} d x \int_{-w / 2}^{w / 2} d y \bar{j}_{x}\left(\vec{r}, \omega^{\prime}\right) \\
& \quad \times \cos \left(\frac{\pi y}{w(z)}\right)
\end{aligned}
$$

where $k_{z}^{2}(z)=(\omega / c)^{2}-(\pi / w)^{2}-\left(w^{\prime} / 2 w\right)^{2}\left(1+\pi^{2} / 3\right)$ and $\bar{j}_{x}\left(\vec{r}, \omega^{\prime}\right)=\pi^{-1} \int_{-\infty}^{\infty} j_{x}(\vec{r}, t) e^{i \omega^{\prime} t} d t$. Here $\bar{j}_{x}\left(\vec{r}, \omega^{\prime}\right)$ is the Fourier amplitude of the current density. We will consider that at the section $z=0$ the FEL amplifier is seeded by the $\mathrm{TE}_{01}$ mode with a frequency of $\omega$ and amplitude $V_{0}$, and the interaction region is ideally matched to a regular output waveguide at the section $z=L$ :

$$
\begin{aligned}
& \left.\left(\frac{\partial \bar{V}}{\partial z}+i k_{z} \bar{V}\right)\right|_{z=0}=2 i k_{z} V_{0} \delta\left[\omega^{\prime}-\omega\right], \\
& \left.\left(\frac{\partial \bar{V}}{\partial z}-i k_{z} \bar{V}\right)\right|_{z=L}=0 .
\end{aligned}
$$

The conditions for the waveguide profile at the ends of the interaction region have the following form: $w^{\prime}(0)=$ $w^{\prime}(L)=0$. Let us perform approximate inverse Fourier transformation [4,32]. We assume that $\bar{j}_{x}\left(\vec{r}, \omega^{\prime}\right)$ is the narrow-band signal with a fundamental frequency of $\omega$. This means that the current density can be written as $j_{x}(\vec{r}, t)=\operatorname{Re}\left\{J_{x}(\vec{r}, t) e^{-i \omega t}\right\}$, where $J_{x}(\vec{r}, t)$ is a slow function of time such that

$$
\begin{aligned}
J_{x}(\vec{r}, t) & \approx \frac{\omega}{\pi} \int_{t-\pi / \omega}^{t+\pi / \omega} j_{x}(\vec{r}, t) e^{i \omega t} d t \\
& \approx \int_{-\infty}^{\infty} \bar{j}_{x}(\vec{r}, \omega+\Delta \omega) e^{-i \Delta \omega t} d(\Delta \omega), \\
\Delta \omega & =\omega^{\prime}-\omega .
\end{aligned}
$$

Expanding $k_{z}^{2}\left(z, \omega^{\prime}\right)$ into Taylor's series over $\omega$ up to the linear term, multiplying Eq. (A3) by $e^{-i \Delta \omega t}$ and integrating it over $\Delta \omega$ from $-\infty$ to $\infty$, we derive the time-dependent excitation equation (3) for the slow-in-time amplitude $V(z, t)=\int_{-\infty}^{\infty} \bar{V}(z, \omega+\Delta \omega) e^{-i \Delta \omega t} d(\Delta \omega)$. The solution (A2) and the boundary conditions (A4) can be rewritten as Eqs. (2) and (4), respectively.

\section{APPENDIX B: NONLINEAR INTERNAL RESONANCES}

It is advanced to divide the Hamiltonian (9) into a nonperturbed (integrable) part that corresponds to the electron motion in the axial homogeneous magnetic field and a small perturbation caused by the undulator magnetic field. Based on the nonperturbed system (the undulator field is absent $B_{u}=0$ ), we introduce the action-angle variables: 


$$
\begin{aligned}
P_{y} & =-\frac{\sqrt{2 \omega_{c} \mathcal{E} I_{c}}}{c} \sin \vartheta_{c}, \quad P_{z}=k_{u} I_{u}, \\
y & =c \sqrt{\frac{2 I_{c}}{\omega_{c} \mathcal{E}}} \cos \vartheta_{c}-\frac{c^{2} P_{x}}{\omega_{c} \mathcal{E}}, \quad z=\frac{\vartheta_{u}}{k_{u}} ;
\end{aligned}
$$

the initial conditions take the following form:

$$
\begin{aligned}
& \left.I_{u}\right|_{t=t_{e}}=\beta_{\|} \mathcal{E} /\left(k_{u} c\right),\left.\quad \vartheta_{u}\right|_{t=t_{e}}=0, \\
& \left.I_{c}\right|_{t=t_{e}}=\varepsilon^{2} \beta_{\|}^{2} \mathcal{E} \cosh ^{2}\left[k_{u} y_{0}\right] /\left(2 \omega_{c}\right),\left.\quad \vartheta_{c}\right|_{t=t_{e}}=\pi,
\end{aligned}
$$

where $\beta_{\|}=V_{\|} / c, \omega_{c}=|e|_{c} B_{\|} / \mathcal{E}$, and $\omega_{u}=k_{u} V_{\|}$are the partial cyclotron and undulator frequencies, respectively; $\varepsilon=\sqrt{2} \omega_{\beta} / \omega_{u}$ is the dimensionless perturbation parameter, $\omega_{\beta}=|e| c B_{u} /(\sqrt{2} \mathcal{E})$ is the betatron frequency. Using (B1) and some algebra we may rewrite (9) as

$$
\overline{\mathcal{H}}=\sqrt{m_{e}^{2} c^{4}+\left(c k_{u} I_{u}\right)^{2}+2 \omega_{c} \mathcal{E} I_{c}+\varepsilon V(\vec{I}, \vec{\vartheta})} .
$$

Here $\varepsilon V(\vec{I}, \vec{\vartheta})$ is the nonintegrable undulator perturbation,

$$
\begin{aligned}
\varepsilon V(\vec{I}, \vec{\vartheta})= & \sum_{n=0}^{2} \sum_{m=0}^{\infty} V_{n, m}\left(I_{c}\right)\left(\cos \left[n \vartheta_{u}+m \vartheta_{c}\right]\right. \\
& \left.+\cos \left[n \vartheta_{u}-m \vartheta_{c}\right]\right)
\end{aligned}
$$

where for odd values of $m$ the coefficient $V_{n, m}$ is

$$
\begin{aligned}
& V_{0, m}=V_{2, m}=-\varepsilon^{2} \beta_{\|}^{2} \mathcal{E}^{2} \sinh [2 A] I_{m}[2 B] / 4, \\
& V_{1, m}=\varepsilon \beta_{\|} \mathcal{E} \sqrt{2 \omega_{c} \mathcal{E} I_{c}} \cosh [A]\left(I_{m+1}[B]+I_{m-1}[B]\right),
\end{aligned}
$$

and for even values of $m$ the coefficient $V_{n, m}$ is

$$
\begin{aligned}
V_{0, m}= & \varepsilon^{2} \beta_{\|}^{2} \mathcal{E}^{2}\left(\delta_{m, 0}+\cosh [2 A] I_{m}[2 B]\left(2-\delta_{m, 0}\right)\right) / 8 \\
V_{2, m}= & V_{0, m} \\
V_{1, m}= & -\varepsilon \beta_{\|} \mathcal{E} \sqrt{2 \omega_{c} \mathcal{E} I_{c}} \sinh [A] \\
& \times\left(I_{m+1}[B]+I_{m-1}[B]\left(1-\delta_{m, 0}\right)\right) .
\end{aligned}
$$

Here, $\quad A=-\left(k_{u} y_{0}+\left(\sqrt{2} \omega_{\beta} / \omega_{c}\right) \cosh \left[k_{u} y_{0}\right]\right)=$ const, $B=c k_{u} \sqrt{2 I_{c} /\left(\omega_{c} \mathcal{E}\right)}, \delta_{m, n}$ is the Kronecker delta, $I_{n}(x)$ is the modified Bessel function of the first kind of order $n$. The equations of motion read

$$
\begin{aligned}
\left\{\begin{array}{c}
\dot{I}_{u} \\
\dot{I}_{c}
\end{array}\right\}= & \sum_{n=0}^{2} \sum_{m=0}^{\infty}\left\{\begin{array}{l}
n \\
m
\end{array}\right\} \frac{V_{n, m}\left(I_{c}\right)}{2 \mathcal{E}} \\
& \times\left(\sin \left[n \vartheta_{u}+m \vartheta_{c}\right] \pm \sin \left[n \vartheta_{u}-m \vartheta_{c}\right]\right), \\
\dot{\vartheta}_{u}= & c^{2} k_{u}^{2} I_{u} / \mathcal{E}, \\
\dot{\vartheta}_{c}= & \omega_{c}+\sum_{n=0}^{2} \sum_{m=0}^{\infty} \frac{\partial V_{n, m}\left(I_{c}\right)}{2 \mathcal{E} \partial I_{c}} \\
& \times\left(\cos \left[n \vartheta_{u}+m \vartheta_{c}\right]+\cos \left[n \vartheta_{u}-m \vartheta_{c}\right]\right) .
\end{aligned}
$$

The equation set (B5) has a lot of internal nonlinear resonances $\left(\vartheta_{u} \approx \pm m \vartheta_{c}, 2 \vartheta_{u} \approx \pm m \vartheta_{c}\right)$ between the undulator and cyclotron degrees of freedom. Actually, the successive iterations give in the zero approximation $\vec{I}^{(0)}=$ $\left.\vec{I}\right|_{t=t_{e}}$ and $\vartheta_{u}^{(0)}=\omega_{u}\left(t-t_{e}\right), \vartheta_{c}^{(0)}=\pi+\omega_{c}\left(t-t_{e}\right)$. One can check that the first approximation leads to $\vec{\vartheta}^{(1)}, \vec{I}^{(1)} \propto$ $e^{i\left(n \omega_{u} \pm m \omega_{c}\right) t} /\left(n \omega_{u} \pm m \omega_{c}\right)$. As a result, the closer is $n \omega_{u} \pm m \omega_{c}$ to zero, the more perturbed dynamics is. Applying the nonlinear resonance technique to (B5) and analyzing each internal nonlinear resonance separately, we can show that $I_{c} \propto \sqrt{\varepsilon}$ in the vicinity of the resonance. Using this estimation we can compare the levels of the dominance of different resonances $(\varepsilon \ll 1, k=$ $1,2,3 \ldots)$ :

$$
\begin{array}{rlrl}
\text { if } \vartheta_{u} \approx(2 k-1) \vartheta_{c} & & \text { then } \varepsilon V \propto \varepsilon^{k / 2+3 / 4} \cosh [A], \\
\text { if } \vartheta_{u} \approx 2 k \vartheta_{c} & & \text { then } \varepsilon V \propto \varepsilon^{k / 2+1} \sinh [A], \\
\text { if } 2 \vartheta_{u} \approx(2 k-1) \vartheta_{c} & \text { then } \varepsilon V \propto \varepsilon^{k / 2+7 / 4} \sinh [2 A], \\
\text { if } 2 \vartheta_{u} \approx 2 k \vartheta_{c} & \text { then } \varepsilon V \propto \varepsilon^{k / 2+2} \cosh [2 A] .
\end{array}
$$

If $\varepsilon$ and $y_{0}$ exceed some thresholds, then there exist regions of chaotic dynamics of the test electron in the phase space. These regions of the phase space correspond to the regions of the nonlinear resonances between degrees of freedom. The most important resonances, in the vicinity of which the onset of the chaos can occur, are $\vartheta_{u} \approx \vartheta_{c}, \vartheta_{u} \approx 2 \vartheta_{c}$, and $\vartheta_{u} \approx 3 \vartheta_{c}$ (see Figs. 2 and 5).

[1] M. A. Agafonov, A. V. Arzhannikov, N. S. Ginzburg, V. G. Ivannenko, P. V. Kalinin, S. A. Kuznetsov, N. Y. Peskov, and S.L. Sinitsky, IEEE Trans. Plasma Sci. 26, 531 (1998).

[2] S. L. Sinitsky, A. V. Arzhannikov, V.T. Astrelin, P. V. Kalinin, and V.D. Stepanov, IEEE Trans. Plasma Sci. 37, 1885 (2009).

[3] G. Dattoli, A. Doria, G. P. Gallerano, L. Giannessi, E. Giovenale, G. Messina, F. Orsitto, A. Renieri, and I. Spassovski, in Proceedings of the 34th International Conference on Infrared, Millimeter, and Terahertz Waves, Busan, Korea, 2009, p. 1-154.

[4] L.A. Vainshtein and V. A. Solntsev, Lectures on Microwave Electronics (Soviet Radio, Moscow, USSR, 1973).

[5] R. C. Davidson, Physics of Nonneutral Plasmas (Imperial College Press, London, UK, 2001).

[6] N. Kroll, P. Morton, and M. Rosenbluth, IEEE J. Quantum Electron. 17, 1436 (1981).

[7] H. P. Freund and S. H. Gold, Phys. Rev. Lett. 52, 926 (1984).

[8] T. J. Orzechowski et al., Phys. Rev. Lett. 57, 2172 (1986).

[9] H. P. Freund and A. K. Ganguly, Phys. Rev. A 33, 1060 (1986). 
[10] J.H. Booske, M.A. Basten, A.H. Kumbasar, T. M. Antonsen, S. W. Bidwell, Y. Carmel, W. W. Destler, and V. L. Granatstein, Phys. Plasmas 1, 1714 (1994).

[11] H. P. Freund, H. Bluem, and C. L. Chang, Phys. Rev. A 36, 2182 (1987).

[12] N. M. Makarov and Y. V. Tarasov, Phys. Rev. B 64, 235306 (2001).

[13] V. A. Goryashko, K. Ilyenko, and A. Opanasenko, Phys. Rev. ST Accel. Beams 12, 100701 (2009).

[14] L. Michel-Lours, A. Bourdier, and J.M. Buzzi, Phys. Fluids B 5, 965 (1993).

[15] C. Chen and R.C. Davidson, Phys. Rev. A 42, 5041 (1990).

[16] H.P. Freund and T.M. Antonsen, Principles of FreeElectron Lasers (Chapman \& Hall, New York, 1995).

[17] P. Sprangle and V.L. Granatstein, Phys. Rev. A 17, 1792 (1978).

[18] M.E. Conde and G. Bekefi, Phys. Rev. Lett. 67, 3082 (1991).

[19] K. Sakamoto, T. Kobayashi, Y. Kishimoto, S. Kawasaki, S. Musyoki, A. Watanabe, M. Takahashi, H. Ishizuka, and M. Shiho, Phys. Rev. Lett. 70, 441 (1993).

[20] V. A. Goryashko, http://arxiv.org/abs/1004.1373 (2010).

[21] R. Bonifacio, R. Corsini, L. D. Salvo, P. Pierini, and N. Piovella, Kivista del Nuovo Cimento 15, 1 (1992).
[22] W. Colson, Phys. Lett. A 59, 187 (1976).

[23] R. Bonifacio, C. Pelligrini, and L. Narducci, Opt. Commun. 50, 373 (1984).

[24] H. P. Freund and A. K. Ganguly, Phys. Rev. A 34, 1242 (1986).

[25] S. Cheng, W. W. Destler, V.L. Granatstein, T. M. Antonsen, B. Levush, J. Rodgers, and Z.X. Zhang, IEEE Trans. Plasma Sci. 24, 750 (1996).

[26] J. Barré, T. Dauxois, G. DeNinno, D. Fanelli, and S. Ruffo, Phys. Rev. E 69, 045501(R) (2004).

[27] S. E. Tsimring, Electron Beams and Microwave Vacuum Electronics (Wiley, New Jersey, 2007).

[28] V.A. Solnzev, Electronic Engineering 11, 87 (1971).

[29] M. Mitchell, An Introduction to Genetic Algorithms (MIT Press, Cambridge, Massachusetts, 1999).

[30] R.D. Richtmyer and K.W. Morton, Difference Methods for Initial-Value Problems (Interscience, New York, 1967).

[31] N.S. Ginzburg, R. M. Rozental, N. Y. Peskov, A. V. Arzhannikov, and S. L. Sinitskii, Phys. Tech. J. 46, 1545 (2001).

[32] D. I. Trubetskov and A. E. Hramov, Lectures on Microwave Electronics for Physicists (Phizmatlit, Moscow, Russia, 2003). 